\title{
Quantum disordered phase in a doped antiferromagnet
}

\author{
C. Kübert and A. Muramatsu \\ Institut für theoretische Physik der Universität Würzburg, Am Hubland, \\ 97074 Würzburg, Germany
}

(December 7, 2017)

\begin{abstract}
A quantitative description of the transition to a quantum disordered phase in a doped antiferromagnet is obtained with a U(1) gauge-theory, where the gap in the spin-wave spectrum determines the strength of the gauge-fields. They mediate an attractive long-range interaction whose possible bound-states correspond to charge-spin separation and pairing.
\end{abstract}

PACS numbers: 71.27.+a, 74.20.Mn, 75.10.Jm

Typeset using REVTEX 
Based on the previous pioneering analysis of the order-disorder transition in a quantum antiferromagnet [1,2], a phase diagram for the high- $\mathrm{T}_{\mathrm{c}}$ cuprates (HTC) was proposed recently [3] that gives a unified view [4] of nuclear relaxation, magnetic susceptibility and neutron scattering experiments, with a spin-gap in the quantum disordered (QD) phase. In spite of these virtues, the connection with the doped materials remains phenomenological and moreover, the question of superconductivity remains unaddressed.

It is shown here, that a) a quantitative description of the transition to the QD phase can be obtained on the basis of a realistic, microscopic model of the HTC, b) the mass-gap of the spin-wave excitations in the QD phase gives the strength of a gauge-field that mediates a long-range interaction among dopant holes and S- $\frac{1}{2}$ magnetic excitations, and c) the possible bound states correspond to charge-spin separation and pairing. Thus, a basis is provided to the phenomenological description of Refs. [3, 4] and the relationship to charge-spin separation and pairing is revealed.

The underlying microscopic model is the spin-fermion (SF) one [5] that results from a strong-coupling expansion of the 3-band Hubbard model [6,7]. It is characterized by a Kondo-like coupling of the dopant holes to localized spins on the $\mathrm{Cu}$-sites with a strength $J_{K}$ and a Heisenberg antiferromagnetic (AF) interaction among the $\mathrm{Cu}$-spins with strength $J_{H}$. The long-wavelength limit of the model assuming short-range AF order is given by [8]:

$$
\begin{aligned}
S & =S_{F}+S_{N L \sigma}, \\
S_{F} & =\int_{0}^{\beta} d \tau \int d^{2} r\left[c^{\dagger} \partial_{\tau} c+\frac{1}{2 m_{r}} \partial_{r} c^{\dagger} \partial_{r} c-\gamma_{r}\left(\partial_{r} \vec{n}\right)^{2} c^{\dagger} c+i \sum_{\mu} \gamma_{\mu} \vec{J}_{\mu}^{S} \cdot \vec{J}_{\mu}^{F}\right] \\
S_{N L \sigma} & =\frac{1}{2 g} \int_{0}^{\beta} d \tau \int d^{2} r\left[\left(\partial_{r} \vec{n}\right)^{2}+\frac{1}{c^{2}}\left(\partial_{\tau} \vec{n}\right)^{2}\right],
\end{aligned}
$$

where $c^{\dagger}=\left(c_{\downarrow}^{\dagger}, c_{\uparrow}^{\dagger}\right)$ is a fermionic spinor and $\vec{n}$ denotes the order parameter of the spin background, whose dynamics is given by the $O(3)$ non-linear $\sigma$ model (3). The first two terms in $S_{F}$ describe the kinetics of the fermions, where for low doping the bare bandstructure is given by an anisotropic effective mass $m_{r}$, with $r=\perp$, $\|$ denoting the directions perpendicular and parallel to the border of the magnetic Brillouin-zone around the points $\vec{k}_{\text {min }}=\left(\frac{\pi}{2}, \pm \frac{\pi}{2}\right)$. Such hole pockets result from the assumed short-range AF order of the 
spin-background [8]. The third term gives a renormalization of the spin-stiffness of the nonlinear $\sigma$ model, whenever the corresponding site is occupied by a hole. The fourth term gives a current-current coupling similar to the one obtained by Shraiman and Siggia for the $t$ - $J$ model [9]. The temporal component $(\mu=\tau)$ corresponds to a coupling of the local spin-density $\vec{J}_{\tau}^{F}=c^{\dagger} \overrightarrow{\sigma c}$ of the fermions to the background magnetization $\vec{J}_{\tau}^{S}=\vec{n} \times \partial_{\tau} \vec{n}$, whereas the spatial components corresponds to the coupling between the spin current of the holes $\vec{J}_{r}^{F}=\left(\partial_{r} c^{\dagger} \vec{\sigma} c-c^{\dagger} \vec{\sigma} \partial_{r} c\right)$ and the magnetization current of the background $\vec{J}_{r}^{S}=\vec{n} \times \partial_{r} \vec{n}$. The connection of the parameters $m_{r}$ and $\gamma_{\mu}$ to the microscopic ones of the SF model and a detailed derivation of the above results is given in Ref. [\&]. For simplicity we assume here isotropic parameters $m_{\perp}=m_{\|}=m$ and $\gamma_{\perp}=\gamma_{\|}=\gamma$ in the following [10]. Further insight is obtained by rotating the fermionic spinor to a spin quantization axis (SQA), which is given by the local direction of the order parameter field $\vec{n}(\vec{r}, \tau)$ :

$$
\begin{aligned}
p(\vec{r}, \tau) & =U^{\dagger}(\vec{r}, \tau) c(\vec{r}, \tau), \\
\sigma^{z} & =U^{\dagger}(\vec{r}, \tau) \vec{\sigma} \cdot \vec{n}(\vec{r}, \tau) U(\vec{r}, \tau),
\end{aligned}
$$

where $U(\vec{r}, \tau)$ is an $S U(2)$ rotation matrix. In the following we use a $C P^{1}$ representation 11] for the rotation matrix $U$ :

$$
U=\left(\begin{array}{cc}
z_{1} & -\bar{z}_{2} \\
z_{2} & \bar{z}_{1}
\end{array}\right)
$$

with $\bar{Z} Z=1, \bar{Z}=\left(\bar{z}_{1}, \bar{z}_{2}\right)$,where $z_{i}$ are complex numbers. The transition from the action in the laboratory reference frame (11) with a fixed SQA to a field theory in the rotating reference frame is achieved by the following set of transformation equations:

$$
\begin{gathered}
\text { rotating SQA } \longleftrightarrow \text { uniform SQA } \\
p_{\mu} \longleftrightarrow c_{\mu} \\
\partial_{\mu}+i A_{\mu} \sigma^{z} \longleftrightarrow \partial_{\mu}-\frac{i}{2} \vec{\sigma} \cdot\left(\vec{n} \times \partial_{\mu} \vec{n}\right) \\
K_{\mu}=i B_{\mu} \sigma^{-}+i \bar{B}_{\mu} \sigma^{+} \longleftrightarrow \frac{i}{2} \vec{\sigma} \cdot\left(\vec{n} \times \partial_{\mu} \vec{n}\right),
\end{gathered}
$$


where we have introduced a composite gauge field $A_{\mu}$ and off-diagonal contributions denoted by $B_{\mu}$ and $\bar{B}_{\mu}$ :

$$
\begin{aligned}
& A_{\mu}=-i \bar{Z} \partial_{\mu} Z \\
& B_{\mu}=i\left(z_{2} \partial_{\mu} z_{1}-z_{1} \partial_{\mu} z_{2}\right)=Z^{T} \sigma^{y} \partial_{\mu} Z, \\
& \bar{B}_{\mu}=i\left(\bar{z}_{1} \partial_{\mu} \bar{z}_{2}-\bar{z}_{2} \partial_{\mu} \bar{z}_{1}\right)=-\bar{Z} \sigma^{y} \partial_{\mu} \bar{Z}^{T},
\end{aligned}
$$

which are related to the $S U(2)$ rotation via $U^{\dagger} \partial_{\mu} U=i \sigma^{z} A_{\mu}+i \bar{B}_{\mu} \sigma^{+}+i B_{\mu} \sigma^{-}$. Therefore we obtain after applying the transformations (7) to the action (1) the following alternative one:

$$
\begin{aligned}
S_{F} & =\int_{0}^{\beta} d \tau \int d^{2} r\left\{p^{\dagger}\left(D_{\tau}^{F}+2 \tilde{\gamma}_{\tau} K_{\tau}\right) p+\frac{1}{2 m} \bar{D}_{r}^{F} p^{\dagger} D_{r}^{F} p\right. \\
& \left.+\tilde{\gamma}\left[2\left(\partial_{r} p^{\dagger} K_{r} p-p^{\dagger} K_{r} \partial_{r} p\right)-4 p^{\dagger} K_{r} K_{r} p\right]\right\}, \\
S_{C P^{1}} & =\frac{2}{g} \int_{0}^{\beta} d \tau \int d^{2} r\left\{\bar{D}_{r}^{B} \bar{Z} D_{r}^{B} Z+\frac{1}{c^{2}} \bar{D}_{\tau}^{B} \bar{Z} D_{\tau}^{B} Z\right\}
\end{aligned}
$$

with $D_{\mu}^{B}=\left(\partial_{\mu}+i A_{\mu}\right)$ and $D_{\mu}^{F}=\left(\partial_{\mu}+i \sigma^{z} A_{\mu}\right)$ denoting covariant derivatives for the bosons and fermions, respectively, and $\tilde{\gamma}_{\tau}=\gamma_{\tau}+1$ and $\tilde{\gamma}=\gamma+1 / 2 m$. The action in the rotating reference frame is invariant under a $U(1)$ transformation with $A_{\mu}$ being the corresponding gauge connection [12]. We would like to stress that the fermionic fields in Eq. (11) are the physical ones and not due to an enlargement of the Hilbert space like in a slave-boson treatment of the $t-J$ model [13]. The gauge fields arise by relating an $S U(2)$ rotation in spin-space and a vector on the sphere $S^{2}$. The manifold $S U(2)$ is isomorphic to $S^{3}$, however, the vector $\vec{n}$ in $S^{2}$ fixes only two of the three angles in $S^{3}$, and hence, a phase remains free.

The dynamics of the gauge fields present in Eqs. (11) and (12) is generated by fluctuations of the $C P^{1}$ non-linear $\sigma$ model as well as by the fermions [13, 14], which can be systematically treated within a large- $N$ expansion [14]. In contrast to earlier calculations on related gauge theories [15], where nondiagonal contributions like the terms $\propto K_{\mu}$ in Eq. (11) were usually omitted, it will be shown that exactly these terms are responsible for the appearance of a doping induced quantum phase transition in this model. 
We proceed further by introducing $L$ copies of $p_{\uparrow}$ and $p_{\downarrow}$ fermions and extend the $C P^{1}$ fields to $C P^{N-1}$ ones, where now $\bar{Z}=\left(\bar{z}_{1}, \ldots, \bar{z}_{N}\right)$ with $N=2 L$. After properly normalizing the action and rescaling the fields the partition function of the field theory given by Eqs. (11) and (12) reads:

$$
\begin{aligned}
& \mathcal{Z}\left[Q_{\mu}\right]=\int \mathcal{D} Z \mathcal{D} \bar{Z} \mathcal{D} p \mathcal{D} p^{\dagger} \prod_{\vec{r}, \tau} \delta\left(\bar{Z} Z-\frac{2 N}{g}\right) \\
& \cdot \exp \left\{-S_{F}-S_{C P^{N-1}}+\int_{0}^{\beta} d \tau \int d^{2} r \frac{g}{2 N} Q_{\mu} A_{\mu}\right\},
\end{aligned}
$$

where we have introduced a source $Q_{\mu}$ for the gauge field $A_{\mu}$, which we use to separate the fermionic contribution to the gauge fields from the one coming from the $C P^{N-1}$ model, by substituting $(g / 2 N) A_{\mu}$ by a functional derivative with respect to the source field $Q_{\mu}$. Integrating out the fermions leads to $S_{F}=-N \operatorname{Tr} \ln \left[\Delta_{F}^{0}+\Delta_{F}^{A}+\Delta_{F}^{B}\right]$, where we have divided the fermionic contribution into three terms, the first one is the free part, the second one contains the gauge field $A_{\mu}$ and the third one contains the off-diagonal $B_{\mu}$ terms :

$$
\begin{aligned}
\Delta_{F}^{0} & =\left(\partial_{\tau}+\frac{1}{2 m} \partial_{r}^{2}\right) \\
\Delta_{F}^{A} & =i \sigma^{z} \frac{\delta}{\delta Q_{\tau}}+i \sigma^{z} \frac{1}{2 m}\left[\left(\partial_{r} \frac{\delta}{\delta Q_{r}}\right)+\frac{\delta}{\delta Q_{r}} \partial_{r}\right]-\frac{1}{2 m} \frac{\delta^{2}}{\delta Q_{r}{ }^{2}} \\
\Delta_{F}^{B} & =i \tilde{\gamma}_{\tau}\left(\frac{g}{2 N}\right) 2\left(B_{\tau} \sigma^{-}+\bar{B}_{\tau} \sigma^{+}\right)+\tilde{\gamma}\left(\frac{g}{2 N}\right) 2\left[\left(B_{r} \sigma^{-}+\bar{B}_{r} \sigma^{+}\right) \overleftrightarrow{\partial_{r}}\right] \\
& +\left(\frac{g}{2 N}\right)^{2} \tilde{\gamma} 4 B_{r} \bar{B}_{r}
\end{aligned}
$$

This division turns out to be useful, since because of the underlying $S U(2)$ structure the $A_{\mu}$ and $B_{\mu}$ terms in $S_{F}$ do not mix and we therefore can treat them independent form each other. We first show that the $B_{\mu}$ terms modify the $C P^{N-1}$ model:

$$
\begin{aligned}
S_{F}^{B_{\mu}}= & \int d^{3} p N\left(\frac{g}{2 N}\right)^{2} 4 \bar{B}_{r}(p) B_{s}(-p) \\
& {\left[\tilde{\gamma} \delta_{r s} \int d^{3} q G_{F}(q)+\frac{1}{2} \tilde{\gamma}^{2} \int d^{3} q G_{F}(q)(2 q+p)_{r}(2 q+p)_{s} G_{F}(q+p)\right] } \\
+ & \int d^{3} p N\left(\frac{g}{2 N}\right)^{2} 4 \bar{B}_{\tau}(p) B_{\tau}(-p) \frac{\tilde{\gamma}_{\tau}^{2}}{2} \int d^{3} q G_{F}(q) G_{F}(q+p)
\end{aligned}
$$

where $G_{F}(q)=1 /\left(i \nu_{n}-\vec{q}^{2} / 2 m\right)$ is the fermionic Greens function and we have introduced $\int d^{3} q$ as a shorthand notation for $1 / \beta \sum_{\nu_{n}} \int d^{2} q /(2 \pi)^{2}$. Here $p=\left(\vec{p}, \omega_{n}\right)$ denotes the wave 
vector together with the Matsubara frequency. Evaluating the integrals in Eq. (17) in the limit $\vec{p} \rightarrow 0$ and $\omega /|\vec{p}| \rightarrow 0$ and noticing that $(g / 2 N) \bar{B}_{\mu} B_{\nu}=\bar{D}_{\mu}^{B} \bar{Z} D_{\nu}^{B} Z$ we obtain for $S_{F}^{B_{\mu}}$ together with the bosonic contribution the following generalized $C P^{N-1}$ model:

$$
S_{C P^{N-1}}^{g e n .}=S_{C P^{N-1}}+S_{F}^{B_{\mu}}=\int_{0}^{\beta} d \tau \int d^{2} r\left\{f_{r}^{\delta} \bar{D}_{r}^{B} \bar{Z} D_{r}^{B} Z+\frac{1}{c^{2}} f_{\tau}^{\delta} \bar{D}_{\tau}^{B} \bar{Z} D_{\tau}^{B} Z\right\}
$$

where we have defined the doping dependent coupling constants $f_{r}^{\delta}=1+2 g \delta\left(\tilde{\gamma}-2 m \tilde{\gamma}^{2}\right)=$ $1-2 g \delta(1+2 \gamma m) \gamma$ and $f_{\tau}^{\delta}=1+2 g c^{2} \tilde{\gamma}_{\tau}^{2} \frac{m}{4 \pi} \theta(\delta)$, and $\theta$ is the usual step-function. As we see from Eq. (18) the $B_{\mu}$ terms lead to coupling constants that are now doping dependent. Before we can integrate out the bosonic variables, we have to decouple the quartic terms. This is achieved by introducing a Hubbard Stratonovich field $\lambda_{\mu}$, which turns out to be equivalent to the $U(1)$ gauge field since it couples linearly to the source field $Q_{\mu}$ :

$$
\begin{aligned}
& \exp \left\{\frac{g}{2 N f_{r}^{\delta}} \int_{0}^{\beta} d \tau \int d^{2} r\left[-\left(\bar{Z} \partial_{\mu} Z\right)\left(\bar{Z} \partial_{\mu} Z\right)+i Q_{\mu}\left(\bar{Z} \partial_{\mu} Z\right)\right]\right\} \\
& =\int \mathcal{D} \lambda_{\mu} \exp \left\{\int_{0}^{\beta} d \tau \int d^{2} r\left[-\frac{2 f_{r}^{\delta}}{g} \lambda_{\mu} \lambda_{\mu}+\frac{1}{\sqrt{N}} \lambda_{\mu} 2 i\left(\bar{Z} \partial_{\mu} Z\right)+\frac{1}{\sqrt{N}} \lambda_{\mu} Q_{\mu}-\frac{g}{8 N f_{r}^{\delta}} Q_{\mu} Q_{\mu}\right]\right\}
\end{aligned}
$$

Here we have rescaled the $Z$ bosons in such a way that the constraint now reads $\bar{Z} Z=$ $2 N f_{r}^{\delta} / g$. We further include this constraint via a Lagrange-multiplier field $\alpha(\vec{r}, \tau)$ into the action and introduce a mass term $M^{2} \bar{Z} Z$, which is arbitrary at this stage. After these modifications the bosonic variables are integrated out and together with the remaining contribution from the fermionic part we get:

$S=N \operatorname{Tr} \ln \Delta_{B}-N \operatorname{Tr} \ln \left(1+\Delta_{F}^{A} / \Delta_{F}^{0}\right)-\int_{0}^{\beta} d \tau \int d^{2} r \frac{i 2 \sqrt{N} f_{r}^{\delta}}{g} \alpha(\vec{r}, \tau)=: \sum_{\nu=1}^{\infty} N^{1-\nu / 2} S^{(\nu)}$,

where

$$
\Delta_{B}=-D_{\mu}^{B} D_{\mu}^{B}+M^{2}-\frac{i \alpha}{\sqrt{N}} .
$$

The covariant derivative is now given by $D_{\mu}^{B}=\partial_{\mu}+i \lambda_{\mu} / \sqrt{N}$ and furthermore the functional derivative with respect to the source $Q_{\mu}$ in $\Delta_{F}^{A}$ is substituted by $\lambda_{\mu} / \sqrt{N}$. In the limit of large- $N$ the following two contributions are important:

$$
S^{(1)}=i \tilde{\alpha}\left[\frac{2 f_{r}^{\delta}}{g}-\int d^{3} q G_{B}(q)\right]
$$


where $G_{B}(p)=1 /\left(\vec{p}^{2}+w_{n}^{2}+M^{2}\right)$ is the bosonic Greens function, and

$$
S^{(2)}=\frac{1}{2} \int d^{3} p\left\{\lambda_{\mu}(p)\left[\Pi_{\mu \nu}^{F}(p)+\Pi_{\mu \nu}^{B}(p)\right] \lambda_{\nu}(-p)-\alpha(p) F(p) \alpha(-p)\right\}
$$

where $F(p)=\int d^{3} q G_{B}(q) G_{B}(q+p)$ and where we have denoted the individual contributions of the bosons and the fermions to the polarization tensor of the gauge field $\lambda_{\mu}$ by $\Pi_{\mu \nu}^{B}(p)$ and $\Pi_{\mu \nu}^{F}(p)$, respectively. At this point we want to emphasize that without taking into account the nondiagonal $K_{\mu}$ terms of the fermionic action (2) there would appear no doping dependent parameter as $f_{r}^{\delta}$ in the expression for $S^{(1)}$.

In the large- $N$ limit, the saddle-point condition $S^{(1)}=0$ has to be imposed [14], as can be seen from Eq. (20) such that a definite relationship between the mass $M$ and the temperature as well as the parameters of the model is established (for $\beta \gg 1$ ):

$$
M=\frac{2}{\beta} \operatorname{arcsinh}\left[\exp \left\{-4 \pi \beta\left(\frac{f_{r}^{\delta}}{g}-\frac{1}{g_{c}}\right)\right\}\right],
$$

where we have defined the critical coupling constant $g_{c}=8 \pi / \Lambda(\Lambda$ is an ultraviolet cutoff $\sim a$, the lattice constant). Eq. (24) is the relationship obtained for the inverse correlation length of the non-linear $\sigma$ model [1, 2], and the same discussion applies here. As pointed out first by Chakravarty et al. [1], three different phases appear depending on whether the coupling constant is smaller, larger, or equal to the critical one.

By introducing the renormalized spin stiffness $\rho=1 / g-1 / g_{c}$ at zero doping, where the system is in the Néel ordered phase (at $T=0$ ) [1], and setting the expression in the exponential of Eq. (24) equal to zero, we obtain the following result for the critical doping:

$$
\delta_{c}=\frac{\rho}{2 \gamma(1+2 \gamma m)} .
$$

An estimation of the critical doping using the parameters $m=3 / 2, \gamma=1 / 12$ and the value $\rho=7.9 \cdot 10^{-3}$ [16] yields $\delta_{c} \approx 3.8 \%$. This is the first central result of this paper. We have also calculated the spin-wave velocity and obtain a reduction by doping to only about $80 \%$ of its undoped value.

The same result as Eq. (24) can be obtained by integrating out the fermions in Eq. (2) similarly to previous calculations on doped antiferromagnets [17. However, in this case 
additional information can be obtained. As can be seen from Eq. (23) a kinetic energy term for the gauge field was dynamically generated. Evaluating the function $F(p)$ and the polarization tensor of the bosons and fermions in the limit of $\vec{p} \rightarrow 0$ and $\omega /|\vec{p}| \rightarrow 0$, we get choosing the Coulomb gauge $\vec{\nabla} \cdot \vec{\lambda}=0$ a massive propagator for both the lagrange multiplier field $\alpha$ and the time component of the gauge field $\lambda_{\tau}$ :

$$
\begin{aligned}
D^{\alpha} & =\frac{1}{8 \pi M} \\
D_{\tau \tau}^{\lambda} & =\frac{\vec{p}^{2}+\omega^{2}}{\vec{p}^{2}} \frac{1}{\frac{1}{24 \pi M}\left(\vec{p}^{2}+\omega^{2}\right)+\frac{m}{2 \pi}}
\end{aligned}
$$

Therefore fluctuations of the constraint field $\alpha$ as well as the fluctuations of the time component of the gauge field $\lambda_{\tau}$ produce only short-range density-density interactions. On the other hand for the propagator of the spatial part of the gauge field $\lambda_{i}$ turns out to be massless:

$$
D_{i j}(\vec{p}, \omega)=\frac{1}{2}\left(\delta_{i j}-\frac{p_{i} p_{j}}{\vec{p}^{2}}\right) \frac{M}{\frac{1}{48 \pi} \vec{p}^{2}-i \rho \frac{M}{m}\left(\frac{\omega}{p v_{F}}\right)} .
$$

This is essentially the same propagator that was found by Nagaosa and Lee [13]. The spatial components of the gauge field produce a long-range current-current interaction between the fermions and the $Z$-bosons. A central difference, however, is given by the fact that the mass $M$ of the spin-excitations determines the strength of the propagator, and hence the strength of the interaction mediated by it. In fact according to the discussion following Eq. (22) the mass $M$, is essentially the gap measured in neutron scattering experiments [4]. Therefore, bound states are only possible in the quantum disordered phase with a finite mass $M$. In this regime the physical spectrum of the theory contains only states with zero charge with respect to the gauge field (singlet states) such as $Z-Z, p-Z$, and $p-p$ bound states. The $Z-Z$ bound states correspond to spin-waves around the antiferromagnetic wavevector, with a gap in the excitation spectrum. The $p$ - $Z$ bound states are spinless charged excitations. Thus, this scenario gives an alternative way to charge-spin separation, where the bare excitations are just spin- $\frac{1}{2}$ fermions but the renormalized ones are spinless. It should be remarked here that in our case charge-spin separation results from an interaction that leads to the 
formation of a bound state and not by spontaneous breaking of gauge-invariance as e.g. in Ref. 13. Finally, the $p-p$ bound state is a singlet with a charge $2 e$ leading to pairing. Hence, charge-spin separation and pairing are intimately connected in our case and result from the same interaction.

In summary, we presented a field-theoretic description of a microscopic model for HTC that explicitly takes into account the influence of doping on the transition to a quantum disordered phase and, moreover, reveals an intimate relationship between the spin-gap, chargespin separation, and pairing.

We acknowledge support by the Deutsche Forschungsgemeinschaft under Project No. Mu 820/5-2. 


\section{REFERENCES}

[1] S. Chakravarty, B.I. Halperin, and D.R. Nelson, Phys. Rev. B39, 2344 (1989).

[2] S. Sachdev and Y. Jinwu, Phys. Rev. Lett. 69, 2411 (1992); A. V. Chubukov and S. Sachdev, Phys. Rev. Lett. 71, 169 (1993).

[3] A. Sokol and D. Pines, Phys. Rev. Lett. 71, 2813 (1993).

[4] V. Barzykin, D. Pines, A. Sokol, and d. Thelen, Phys. Rev. B49, 1548 (1994).

[5] P. Prelovšek, Phys. Lett. A 126, 287 (1988); J. Zaanen and A.M. Olés, Phys. Rev. B37, 4923 (1988); A. Muramatsu, R. Zeyher, and D. Schmeltzer, Europhys. Lett. 7, $473(1988)$.

[6] V.J. Emery, Phys. Rev. Lett. 58, 2794 (1987).

[7] C.M. Varma, Schmitt-Rink, and E. Abrahams, Solid State Commun. 62, 681 (1987).

[8] C. Kübert and A. Muramatsu, Phys. Rev. B47, 787 (1993).

[9] B.I. Shraiman and E.D. Siggia, Phys. Rev. Lett. 61, 467 (1988); Phys. Rev.B 41, 350 (1990); ibid. 42, 2485 (1990).

[10] A general treatment will be given elsewhere. However, this situation is closely given by realistic parameters of the microscopic model [16].

[11] R. Rajaraman, Solitons and instantons (North-Holland, Amsterdam, 1987).

[12] Y. -S. Wu and A. Zee, Phys. Lett. 147 B, 325 (1984).

[13] N. Nagaosa and P.A. Lee, Phys. Rev. Lett. 64, 2450 (1990).

[14] A. D’Adda, M. Lüscher, and P. Di Vecchia, Nucl. Phys. B146, 63 (1978).

[15] X.G. Wen, Phys. Rev. B39, 7223 (1989).

[16] For this estimation we use the following microscopic parameters of the spin-fermion 
model, for which the fermionic mass $m$ is isotropic [8]: $J_{k}=3 / 4, \bar{t}=-0.5$ and $\tilde{t}=$ $-1 / 8$. All parameters are given in units of the hopping between the $\mathrm{Cu}$ and $\mathrm{O}$ orbitals $t_{p d}=1.3 \mathrm{eV}$ of the original 3-band Hubbard model.

[17] A. Muramatsu and R. Zeyher, Nucl. Phys. B346, 387 (1990); A. Muramatsu, Phys. Rev. Lett. 65, 2909 (1990). 